\title{
Etude de la pression exercée par un champ magnétique sur un conducteur plan. Déformations produites $(*)$
}

\author{
R. Chéron et M. Leroy \\ Laboratoire de Physique du Métal, Ecole Nationale Supérieure de Mécanique, 1, rue de la Noë, 44072 Nantes Cedex, France
}

(Reçu le 16 juillet 1980, révisé le 21 novembre 1980, accepté le 26 novembre 1980)

\begin{abstract}
Résumé. - Les efforts exercés sur une plaque métallique par des impulsions magnétiques sont mesurés au moyen d'un pendule balistique. Le calcul de la pression en fonction de l'épaisseur de la plaque nécessite la connaissance précise des caractéristiques de la décharge : amplitude, fréquence, constante de temps. L'inductance de l'ensemble bobine-plaque est le paramètre déterminant.

Les déformations produites varient linéairement selon l'énergie de la décharge.
\end{abstract}

\begin{abstract}
Forces on a metallic plate produced by magnetic pulses are measured with a ballistic pendulum. The pressure calculation needs the knowledge of the electric discharge characteristics : amplitude, frequency, time-constant. The effective inductance of the plate-coil system is the most significant parameter.

The resulting strains are linear function of the stored energy.
\end{abstract}

1. Introduction. - On peut faire passer dans une bobine un courant suffisamment intense pour que le champ produit puisse déformer un échantillon métallique. Sur ce principe a été mis au point, depuis plus de vingt ans, un procédé de mise en forme connu industriellement sous le nom de formage électromagnétique [1].

En pratique, l'énergie nécessaire est stockée dans un condensateur chargé sous une tension de l'ordre de 3 à $20 \mathrm{kV}$, puis libérée brusquement dans une bobine de quelques spires seulement. Le circuit $R, L, C$ ainsi constitué est parcouru par un courant sinusoïdal amorti dont l'intensité peut atteindre $10^{5} \mathrm{~A}$. La pression produite sur un conducteur voisin est due à la répulsion entre le courant traversant la bobine et les courants de Foucault induits dans ce conducteur.

La brièveté de l'impulsion électrique, moins d'une milliseconde, et l'absence de contact entre pièce et poinçon, confèrent à ce procédé des caractéristiques originales sans équivalent dans certains cas [1]. En effet, aux grandes vitesses de déformation, les métaux acquièrent un comportement visqueux $[2,3,4]$ et voient leurs limites de formabilité augmentées [5].

L'obtention des lois de comportement dynamique

(*) Travaux effectués en partie avec l'aide de la D.G.R.S.T. (contrat 77-7-1777). montre ce qu'il est possible de mesurer avec un chargement plus complexe : bobine en forme de spirale et échantillon plan (Fig. 1).

Les auteurs qui ont étudié la mise en forme électromagnétique se sont intéressés à l'expansion et à la compression de tubes $[6,7,8]$ plutôt qu'au placage [9]. des métaux en torsion, traction, compression, constitue l'objectif principal de nos travaux. La présente étude

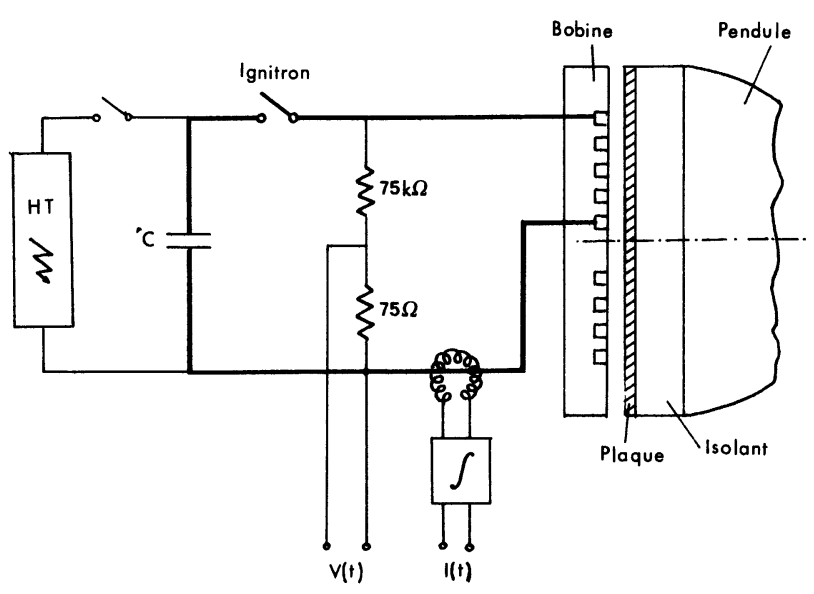

Fig. 1. - Schéma de principe électrique et configuration du placage. [Schematic representation of the experimental arrangement.] 
D'une manière générale, ils ne considèrent pas l'établissement au cours du temps de la pression dans l'épaisseur de l'échantillon et ne mesurent pas les forces appliquées.

Nous envisageons ici le calcul de la pression exercée sur une plaque en tenant compte de la vitesse finie de l'onde électromagnétique et nous comparons le résultat aux déterminations de pression qu'on peut faire par mesure de l'impulsion communiquée à un pendule.

Monté sur une matrice torique (Fig. 2), l'échantillon soumis à cette pression prend une forme en dôme. Il s'agit alors d'un emboutissage électromagnétique. Il est important, en vue d'applications, de connaître à l'avance, même approximativement, l'énergie à prévoir pour obtenir une déformation donnée. Les mesures effectuées donnent cette possibilité pour un diamètre de $100 \mathrm{~mm}$.

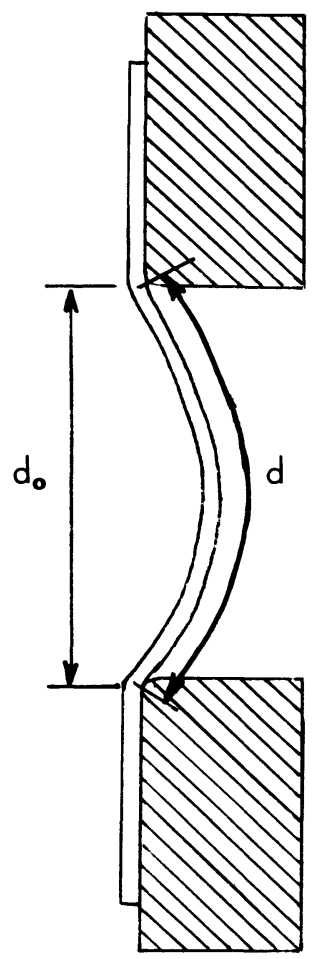

Fig. 2. - Mesure des déformations : $\varepsilon=\log d / d_{0}$.

[Strain measurement $: \varepsilon=\log d / d_{0}$ ]

2. Calcul de l'impulsion de la pression magnétique. 2.1 EXPRESSION DU CHAMP MAGNÉTIQUE DANS LE CONDUCTEUR. - Le courant de décharge d'un condensateur de capacité $C$ chargé sous la tension $V_{0}$ dans une bobine de résistance $R$ et d'inductance $L$ a l'expression :

$$
\begin{array}{ll}
I=0 & t<0 \\
I=I_{0} \mathrm{e}^{-t / \tau} \sin \omega t & t>0
\end{array}
$$

où

$$
\tau=\frac{2 L}{R} \quad \omega^{2}+\frac{1}{\tau^{2}}=\frac{1}{L C} \quad I_{0}=V_{0} \sqrt{\frac{C}{L}} .
$$

Le champ magnétique produit dans l'air par ce courant a la même variation temporelle. Pour une onde électromagnétique plane arrivant sous incidence normale à la surface d'un conducteur (plan $y, z$ ) $H$ ne dépend que de $x$ et de $t$ et est solution de l'équation de diffusion :

$$
\frac{\partial^{2} H}{\partial x^{2}}=\mu \sigma \frac{\partial H}{\partial t} .
$$

Avec la condition à la surface :

$$
H(0, t)=H_{0} \mathrm{e}^{-t / \tau} \sin \omega t \quad t>0 .
$$

Cette équation admet (voir annexe) la solution :

$$
H(x, t)=H_{0} \mathrm{e}^{-x / \delta} \mathrm{e}^{-t / \tau} \sin (\omega t-\eta x)
$$

où

et

$$
\begin{gathered}
\delta=\left(\frac{2}{\mu \sigma \omega}\right)^{1 / 2}\left[\left(1+\frac{1}{\omega^{2} \tau^{2}}\right)^{1 / 2}-\frac{1}{\omega \tau}\right]^{1 / 2} \\
\eta=\left(\frac{2}{\mu \sigma \omega}\right)^{-1 / 2}\left[\left(1+\frac{1}{\omega^{2} \tau^{2}}\right)^{1 / 2}+\frac{1}{\omega \tau}\right]^{-1 / 2}
\end{gathered}
$$

sont respectivement l'épaisseur de peau et la pulsation spatiale en régime transitoire [6].

2.2 Etablissement de la PRession. - Un élément de conducteur d'épaisseur $\mathrm{d} x$ et de section $\mathrm{d} y \mathrm{~d} z$ est soumis à la force de Laplace :

$$
\mathrm{d} F=\mu H \frac{\partial H}{\partial x} \mathrm{~d} x \mathrm{~d} y \mathrm{~d} z
$$

d'où la pression élémentaire :

$$
\mathrm{d} P=\mu H \frac{\partial H}{\partial x} \mathrm{~d} x .
$$

L'expression (2.5) montre que le champ créé à la surface à l'instant $t$ se retrouve, affaibli par un facteur $\mathrm{e}^{\eta x}$, à la profondeur $x$ à l'instant $t+\frac{\eta}{\omega} x$. La vitesse $v=\omega / \eta$ ainsi définie est très faible (de l'ordre de $40 \mathrm{~m} / \mathrm{s}$ ) et on doit en tenir compte dans l'intégration de $\mathrm{d} P$ sur $x$.

En effet, si l'on désigne par $e$ l'épaisseur d'une lame à faces parallèles de conducteur, pour $0<t<e / v$ la pression élémentaire n'est à intégrer que de 0 à $v t$, région effectivement atteinte par le champ. On a ainsi :

$$
\begin{array}{ll}
P_{0}(t)=\int_{0}^{v t} \mathrm{~d} P & 0<t<\frac{e}{v} \\
P_{1}(t)=\int_{0}^{e} \mathrm{~d} P-\int_{e}^{v t} \mathrm{~d} P & \frac{e}{v}<t<\frac{2 e}{v} \\
P_{2}(t)=\int_{0}^{e} \mathrm{~d} P-\int_{e}^{2 e} \mathrm{~d} P+\int_{2 e}^{v t} \mathrm{~d} P & \frac{2 e}{v}<t<\frac{3 e}{v}
\end{array}
$$

etc. 
à cause des réflexions (avec un coefficient très voisin de un) à l'interface métal-air.

2.3 Calcul de L'Impulsion. - La pression $P(t)$ agissant sur la surface $S$ produit une impulsion

$$
\mathfrak{J}=S \int_{0}^{\infty} P(t) \mathrm{d} v
$$

qui se décompose en :

$$
\begin{aligned}
J=S \int_{0}^{\frac{e}{v}} P_{0}(t) \mathrm{d} t+ & S \int_{\frac{e}{v}}^{\frac{2 e}{v}} P_{1}(t) \mathrm{d} t+ \\
& +S \int_{\frac{2 e}{v}}^{3 e v} P_{2}(t) \mathrm{d} t+\cdots
\end{aligned}
$$

Après réaménagement, la totalité des termes s'écrit :

$$
\begin{aligned}
J=S \sum^{\infty}(-1)^{n} & \int_{(n+1) \frac{e}{v}}^{\infty} \mathrm{d} t \int_{n e}^{(n+1) e} \mathrm{~d} P+ \\
& +S \sum^{\infty}(-1)^{n} \int_{n \frac{e}{v}}^{(n+1) \frac{e}{v}} \mathrm{~d} t \int_{n e}^{v t} \mathrm{~d} P .
\end{aligned}
$$

Le calcul de cette double série conduit en tenant compte des ordres de grandeur de certains termes $(\omega \tau>1)$ à

$$
\begin{gathered}
J=S P_{0} \frac{\tau}{4} \frac{1-\exp \left[-2 \eta e\left(1+\frac{1}{\omega \tau}\right)\right]}{1+\exp \left[-2 \eta e\left(1+\frac{1}{\omega \tau}\right)\right]} \\
P_{0}=\frac{\mu H_{0}^{2}}{2}
\end{gathered}
$$

est la pression magnétique pour un milieu infini et un champ sinusoïdal [10].

2.4 Evaluation De $P_{0}$. - L'expression (2.12) de l'impulsion communiquée à une plaque peut se comparer aux résultats expérimentaux en valeur relative (sans connaître $P_{0}$ ).

Si l'on veut une comparaison en vraie grandeur, il faut déterminer localement $H_{0}(y, z)$ et l'intégrer dans le plan de la plaque de manière à en évaluer une valeur moyenne qui soit l'amplitude des ondes planes que nous avons considérées.

Il est plus commode de tirer une valeur globale de $P_{0}$ à partir de la loi de variation de la force en fonction de la distance bobine-plaque $d$ (Smith [11]).

$$
F=\frac{1}{2} I^{2}\left(\frac{\partial L}{\partial d}\right)
$$

où $L$ est l'inductance de l'ensemble bobine-plaque.
3. Dispositif expérimental. - 3.1 MesURE DES PARAMÈTRES ÉLECTRIQUES. - Les caractéristiques de la décharge $I(t)=I_{0} \mathrm{e}^{-t / \tau} \sin \omega t$ sont $I_{0}, \omega$ et $\tau$. Pour les déterminer, nous enregistrons, au moyen d'un oscilloscope numérique, la tension $V(t)$ aux bornes de la bobine et le courant $I(t)$ qui la traverse mesuré par une bobine de Rogowski et un intégrateur analogique.

L'inductance s'obtient alors par

$$
L C=\left(\omega^{2}+\frac{1}{\tau^{2}}\right)^{-1}
$$

et la résistance par

$$
R=\frac{2 L}{\tau} .
$$

3.2 Mesure De L'IMPULSION. - La mesure directe de la pression est difficile puisqu'il s'agit d'un choc s'exerçant sur une surface de l'ordre de $160 \mathrm{~cm}^{2}$. Ces deux exigences semblent hors de portée des capteurs disponibles. Nous avons préféré mesurer l'impulsion totale communiquée à la plaque en la rendant solidaire d'un pendule balistique. Celui-ci est un solide massif d'une masse très supérieure à celle de l'échantillon $(29,8 \mathrm{~kg})$ suspendu librement et sans frottement; sa période est $T_{0}$.

Sous l'action de l'impulsion $\mathfrak{J}$ il décrit une trajectoire circulaire de corde $y$ :

$$
\mathfrak{J}=2 \pi M y / T_{0}
$$

et d'après $(2.12)$ et $(2.13)$ :

$$
P_{\mathrm{m}}=4 \mathrm{~J} / \mathrm{S} \tau .
$$

On trouvera plus de détails sur la constitution du dispositif et des échantillons dans un précédent article [12].

3.3 Mesure Des DÉFormations. - Soient $d_{0}$ le diamètre d'un cercle tracé sur l'échantillon et $d$ la longueur moyenne des méridiennes du dôme de l'échantillon déformé (Fig. 2).

Nous prenons :

$$
\varepsilon=\log \frac{d}{d_{0}} .
$$

4. Résultats expérimentaux. - Les mesures d'impulsion en fonction de l'épaisseur ont été faites sur des plaques d'aluminium (A5), de cuivre et de laiton de pureté commerciale à différentes énergies sur les deux générateurs à notre disposition :

1) Générateur « $6 \mathrm{~kJ} »: 180 \mu \mathrm{F}, 9500 \mathrm{~V}$.

2) Générateur « $12 \mathrm{~kJ} »: 360 \mu \mathrm{F}, 8500 \mathrm{~V}$.

Pour mettre sur le même graphique les résultats obtenus avec les deux générateurs, on a porté en abscisse l'épaisseur réduite $e / \delta$ (Figs. 3,4 et 5 ). 


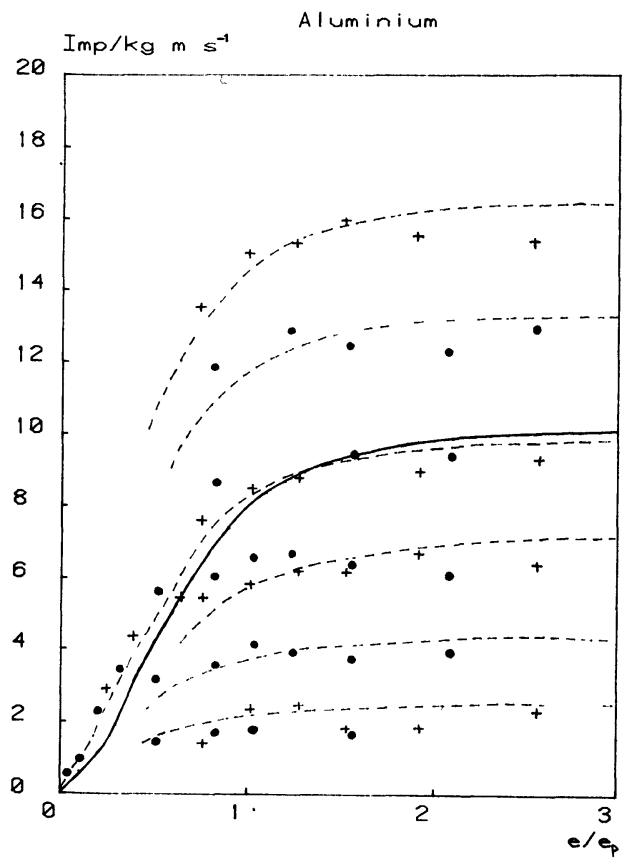

Fig. 3. - Impulsion en fonction de l'épaisseur réduite $e / \delta:+$ générateur $« 6 \mathrm{~kJ} »$, générateur « $12 \mathrm{~kJ} »$; aluminium, en trait plein, courbe calculée pour $6 \mathrm{~kJ}$.

[Momentum versus reduced thickness $e / \delta:+« 6 \mathrm{~kJ} »$ generator, - « $12 \mathrm{~kJ}$ " generator; aluminium, solid line theoretical curve calculated for $6 \mathrm{~kJ}$.]

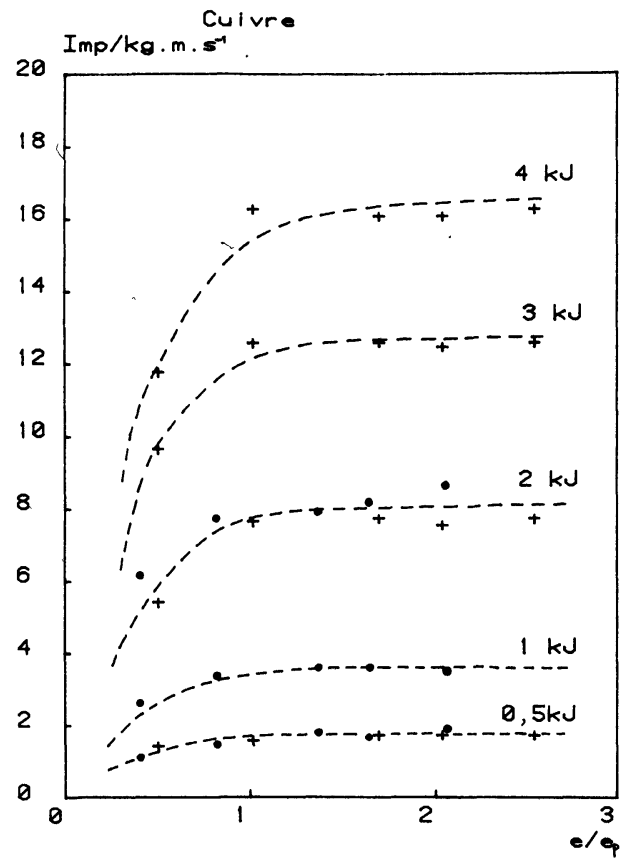

Fig. 4. - Impulsion en fonction de l'épaisseur réduite $e / \delta:+$ générateur « $6 \mathrm{~kJ}$ », générateur « $12 \mathrm{~kJ} »$; cuivre.

[Momentum versus reduced thickness $e / \delta:+\| 6 \mathrm{~kJ}$ » generator, - « $12 \mathrm{~kJ}$ » generator ; copper.]

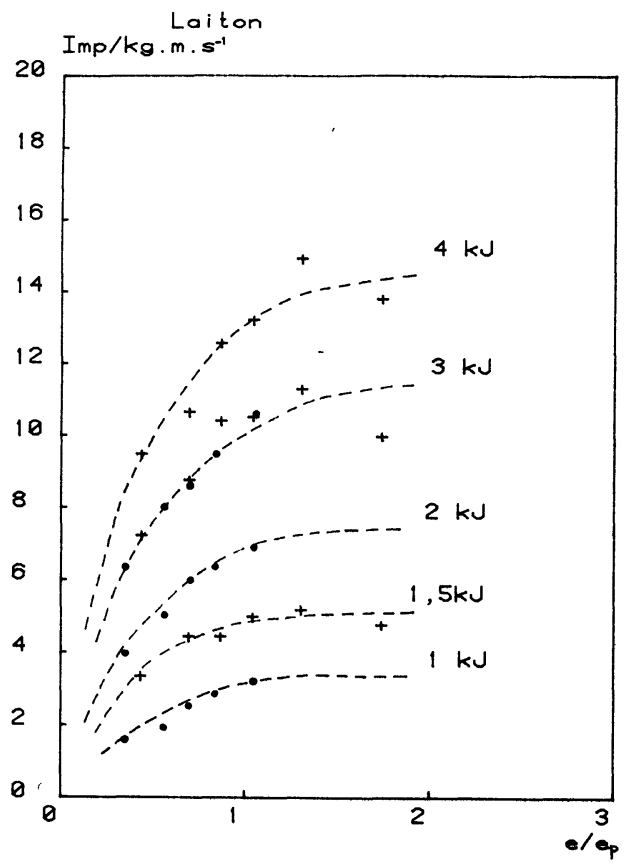

Fig. 5. - Impulsion en fonction de l'épaisseur réduite $e / \delta:+$ générateur « $6 \mathrm{~kJ}$ », - générateur « $12 \mathrm{~kJ}$ »; laiton.

[Momentum versus reduced thickness $e / \delta:+« 6 \mathrm{~kJ}$ » generator, - « $12 \mathrm{~kJ} »$ generator; brass.]

L'application de la formule (2.12) nécessite la connaissance de $\omega$ et $\tau$ qui ne sont pas caractéristiques d'un générateur $(C)$ et d'une bobine donnée $\left(L_{1}, R_{1}\right)$. L'échantillon modifie en effet par mutuelle induction la résistance et l'inductance vues du générateur. On a donc déterminé $\omega$ et $\tau$ en fonction de l'épaisseur de l'échantillon (Figs. 6 et 7 pour l'aluminium). La variation de $L$ et $R$ modifie en outre l'amplitude $I_{0}$ du courant de décharge (Fig. 8).

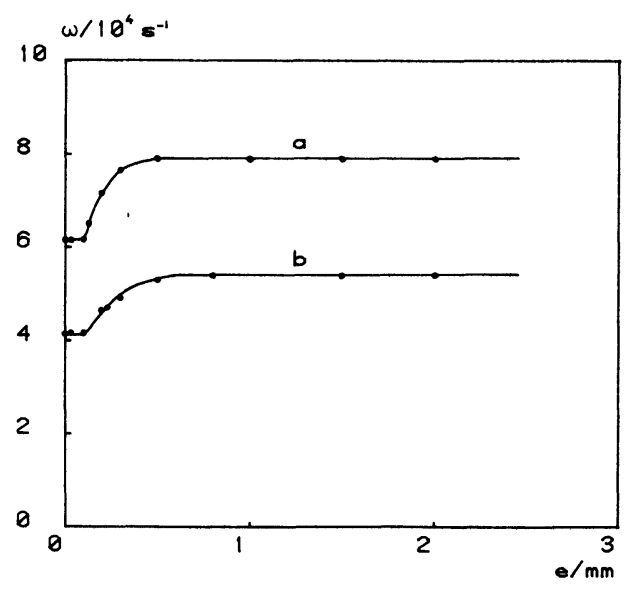

Fig. 6. - Pulsation de la décharge en fonction de l'épaisseur de l'échantillon. Courbe $a$ : générateur « $6 \mathrm{~kJ}$ ». Courbe $b$ : générateur « $12 \mathrm{~kJ}$ ».

[Discharge frequency versus specimen thickness. $a: « 6 \mathrm{~kJ}$ » generator $b: \ll 12 \mathrm{~kJ}$ » generator.] 


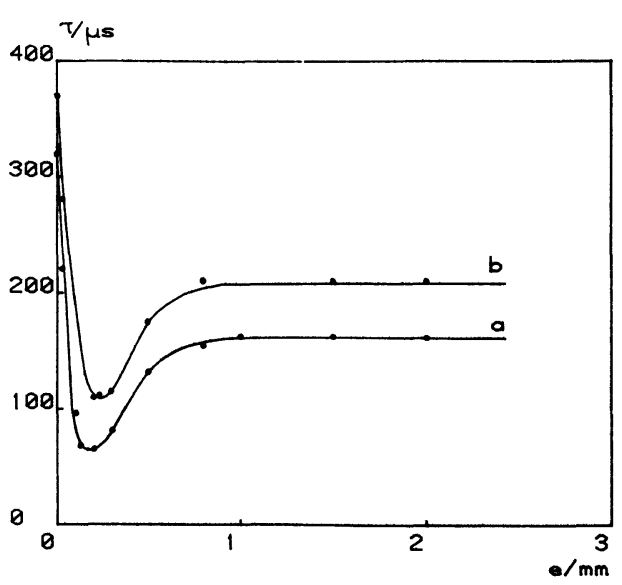

Fig. 7. - Constante de temps de la décharge en fonction de l'épaisseur de l'échantillon. Courbe $a$ : générateur « $6 \mathrm{~kJ}$ ». Courbe $b$ : générateur « $12 \mathrm{~kJ}$ ».

[Time constant versus specimen thickness. $a: « 6 \mathrm{~kJ}$ » generator. $b:$ « $12 \mathrm{~kJ} »$ generator.]

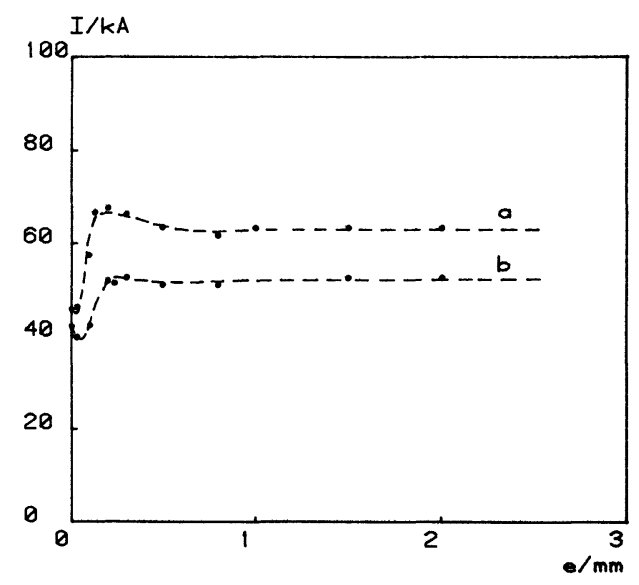

Fig. 8. - Amplitude $I_{0}$ du courant de décharge en fonction de l'épaisseur de l'échantillon. Courbe $a$ : générateur « $6 \mathrm{~kJ}$ ». Courbe $b:$ générateur « $12 \mathrm{~kJ}$ ».

[Discharge current amplitude $I_{0}$ versus specimen thickness. $a$ : « $6 \mathrm{~kJ}$ » generator. $b: \ll 12 \mathrm{~kJ}$ » generator.]

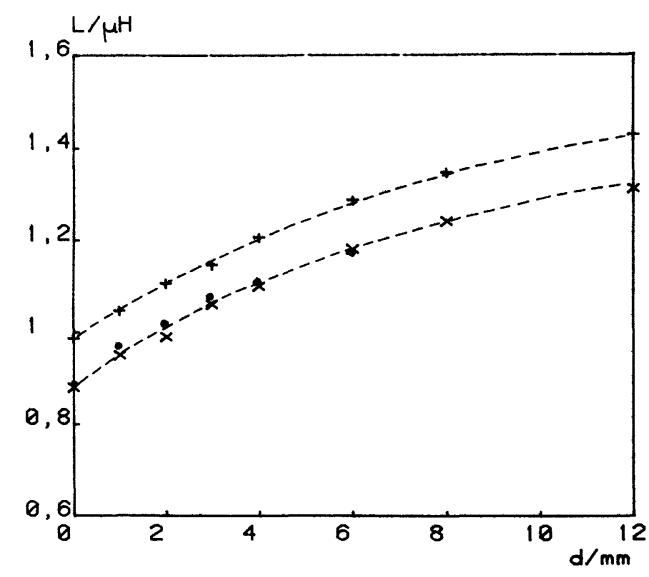

Fig. 9. - Variation de l'inductance selon la distance bobine-plaque :

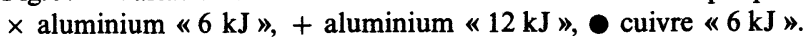

[Inductance variation according to plate-coil distance : $\times$ aluminium « $6 \mathrm{~kJ} »,+$ aluminium « $12 \mathrm{~kJ} », \bullet$ copper $« 6 \mathrm{~kJ} »$.]

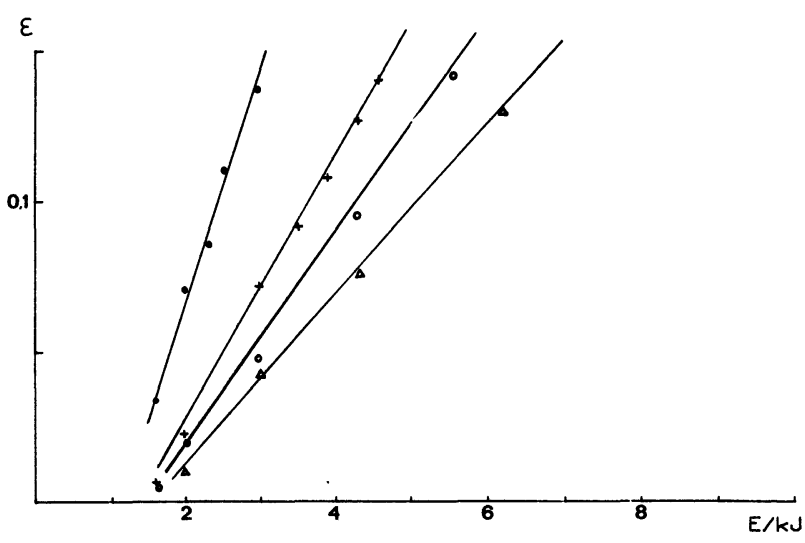

Fig. 10. - Déformation en fonction de l'énergie de la décharge : aluminium : $0,6 \mathrm{~mm} ;+1 \mathrm{~mm} ; 01,2 \mathrm{~mm} ; \triangle 1,5 \mathrm{~mm}$.

[Strain versus discharge energy : aluminium : $0.6 \mathrm{~mm},+1 \mathrm{~mm}$, ○ $1.2 \mathrm{~mm}, \triangle 1.5 \mathrm{~mm}$.]

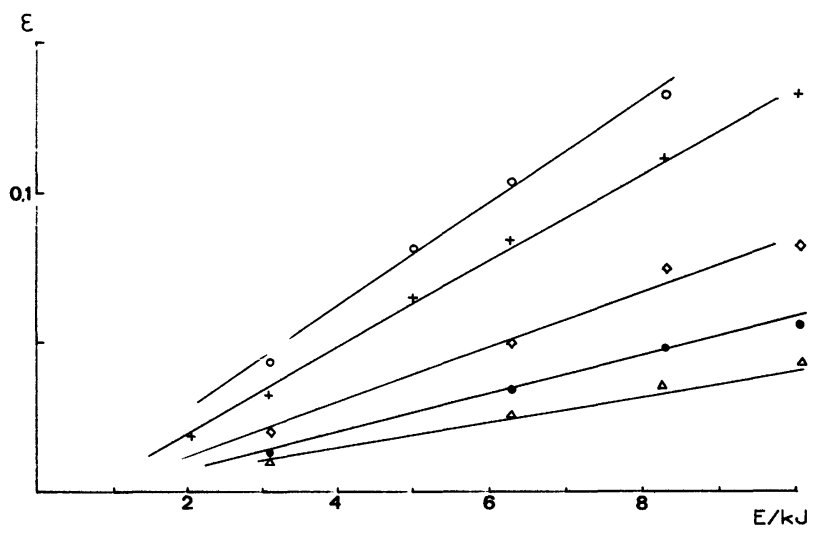

Fig. 11. - Déformation en fonction de l'énergie de la décharge : cuivre : $\bigcirc 0,6 \mathrm{~mm} ;+0,8 \mathrm{~mm} ; \diamond 1 \mathrm{~mm} ; \bullet 1,2 \mathrm{~mm} ; \Delta 1,5 \mathrm{~mm}$.

[Strain versus discharge energy : copper : $00.6 \mathrm{~mm},+0.8 \mathrm{~mm}$, $\diamond 1 \mathrm{~mm}, \bullet 1.2 \mathrm{~mm}, \triangle 1.5 \mathrm{~mm}$.]

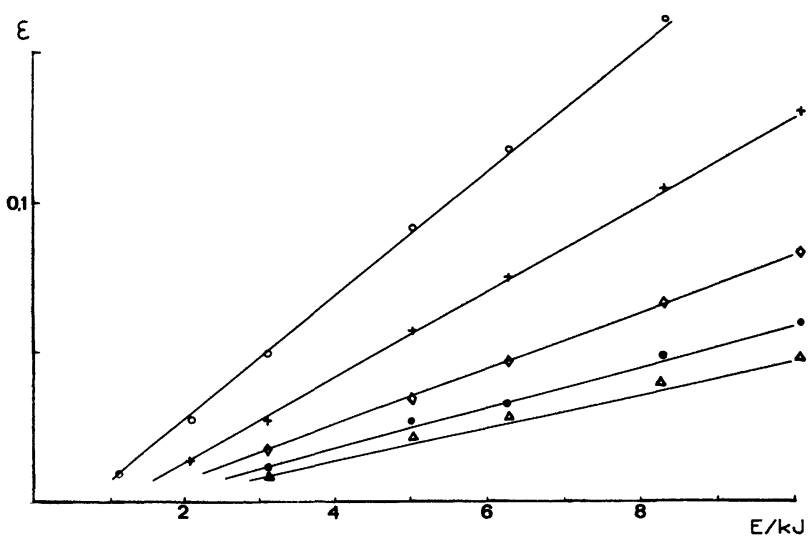

Fig. 12. - Déformation en fonction de l'énergie de la décharge : laiton : $\bigcirc 0,5 \mathrm{~mm} ;+0,8 \mathrm{~mm} ; \diamond 1 \mathrm{~mm} ; \bullet 1,2 \mathrm{~mm} ; \Delta 1,5 \mathrm{~mm}$.

[Strain versus discharge energy : Brass : $00.5 \mathrm{~mm},+0.8 \mathrm{~mm}$, $\diamond 1 \mathrm{~mm}, \bullet 1.2 \mathrm{~mm}, \Delta 1.5 \mathrm{~mm}$.] 
La figure 9 montre la variation de l'inductance équivalente en fonction de la distance bobine-plaque. La formule (2.14) utilise la pente à l'origine de cette courbe qui varie peu d'un métal à l'autre ou d'un générateur à l'autre.

Les déformations ont été mesurées sur les mêmes métaux avec le générateur « $12 \mathrm{~kJ}$ » et une matrice de diamètre $d_{0}=100 \mathrm{~mm}$. Les valeurs obtenues ont été portées en fonction de l'énergie du générateur, à épaisseur constante. De cette manière, ce sont des droites qu'il est facile d'utiliser en vue d'applications (Figs. 10, 11, 12).

5. Analyse des résultats et discussion. -5.1 PARAMÈTRES ÉLECTRIQUES. - Les variations de $\omega$ et $\tau$ en fonction de l'épaisseur des échantillons sont importantes et traduisent une grande variation de $R$ (Fig. 13)

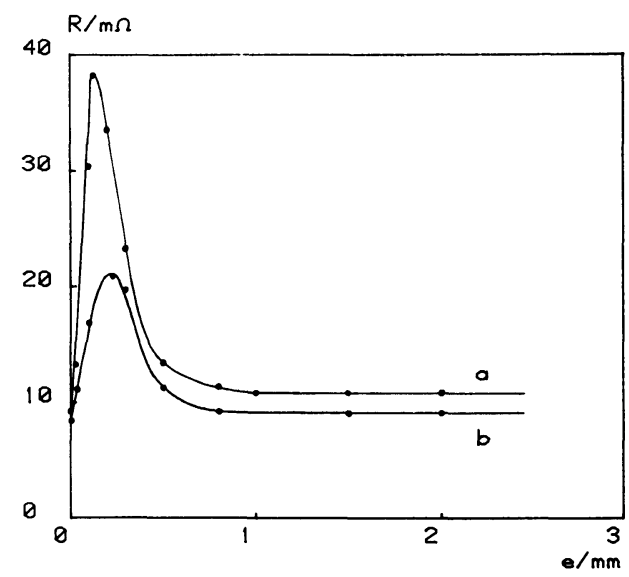

Fig. 13. - Résistance mesurée de l'ensemble bobine-plaque en fonction de l'épaisseur de la plaque : $a$ : générateur « $6 \mathrm{~kJ} » . b$ : générateur « $12 \mathrm{~kJ}$ ».

[Measured electrical resistance of the plate-coil system versus plate thickness : $a:$ « $6 \mathrm{~kJ} »$ generator. $b: \ll 12 \mathrm{~kJ} »$ generator.

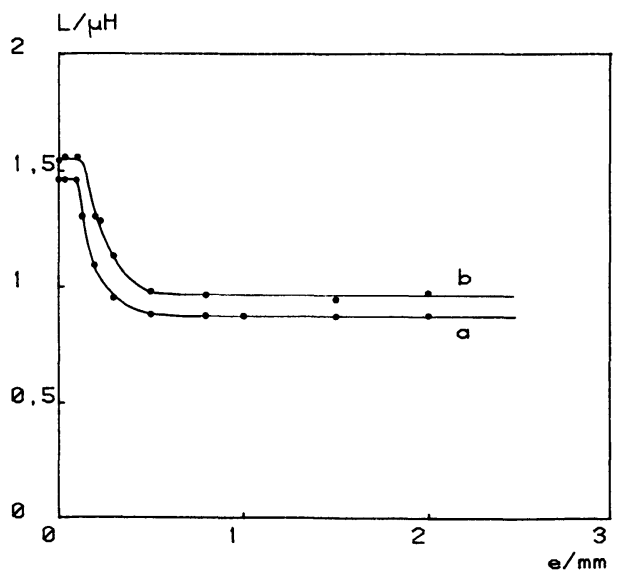

Fig. 14. - Inductance mesurée de l'ensemble bobine-plaque en fonction de l'épaisseur de la plaque : $a$ : générateur « $6 \mathrm{~kJ} » . b$ : générateur « $12 \mathrm{~kJ} »$.

[Measured inductance of the plate-coil system versus plate thickness : $a:$ « $6 \mathrm{~kJ}$ » generator. $b: \ll 12 \mathrm{~kJ}$ » generator.] et de $L$ (Fig. 14) résistance et inductance de l'ensemble bobine-plaque.

$\mathrm{Si}$ nous désignons par $R_{2}$ et $L_{2}$ la résistance et l'inductance de la plaque et par $M$ le coefficient de mutuelle induction, nous avons :

$$
\begin{aligned}
& R=R_{1}+R_{2} M^{2} \omega^{2} /\left(R_{2}^{2}+L_{2}^{2} \omega^{2}\right) \\
& L=L_{1}-L_{2} M^{2} \omega^{2} /\left(R_{2}^{2}+L_{2}^{2} \omega^{2}\right) .
\end{aligned}
$$

Il s'agit alors de concevoir comment varient $R_{2}$ et $L_{2}$ en fonction de l'épaisseur. Lorsqu'on examine une plaque ayant subi la pression magnétique, on $y$ distingue en relief le dessin de la bobine, c'est signe que la majeure partie des courants de Foucault circule dans la plaque suivant une spirale symétrique de celle de la bobine. La self propre de la plaque et le coefficient de couplage dépendant essentiellement du nombre de spires, on peut admettre en première approximation que $L_{2}$ et $M$ ne varient pas en fonction de l'épaisseur. Pour ce qui est de la résistance de la plaque, elle est inversement proportionnelle à l'épaisseur pour $e<\delta$ mais ne tend pas vers zéro quand $e$ augmente à cause de l'effet de peau.

En adoptant $R_{2}=a+b / e$, modèle le plus simple traduisant les considérations précédentes, on obtient les variations de $R$ et $L$ représentées figures 15 et 16 . Ces variations ne sont pas tout à fait les mêmes que celles mesurées (Figs. 13 et 14) mais l'allure générale est respectée, ce qui permet d'affirmer que la résistance de la plaque $R_{2}$ est la principale responsable de l'importante variation de $L$ et surtout de $R$ en fonction de $e$.

5.2 IMPULSION. - Les impulsions mesurées sont celles d'une force; pour les convertir en pression, il faut connaître l'aire sur laquelle cette force agit. Or, cette aire n'est pas parfaitement définie, la zone d'action de la bobine n'ayant pas de frontières nettes. On a donc présenté les résultats avec une dimension

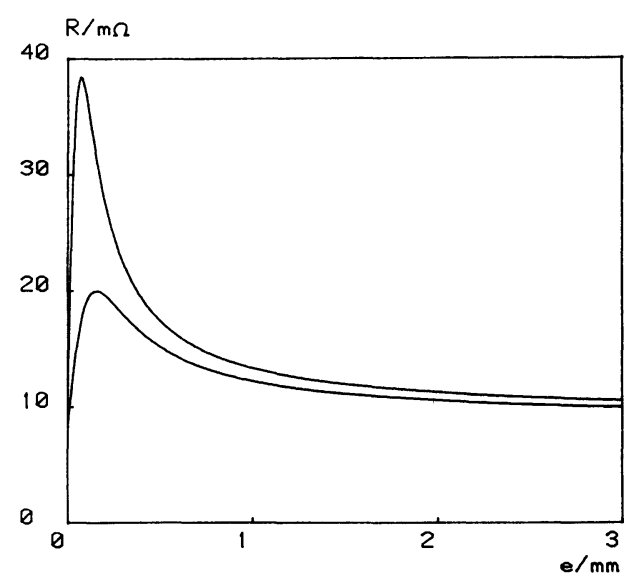

Fig. 15. - Résistance équivalente calculée avec $R_{2}=a+b / e$, $L_{2}=$ Cte, $M=$ Cte.

[Equivalent resistance calculated with $R_{2}=a+b / e, L_{2}=$ Cte, $M=$ Cte.] 


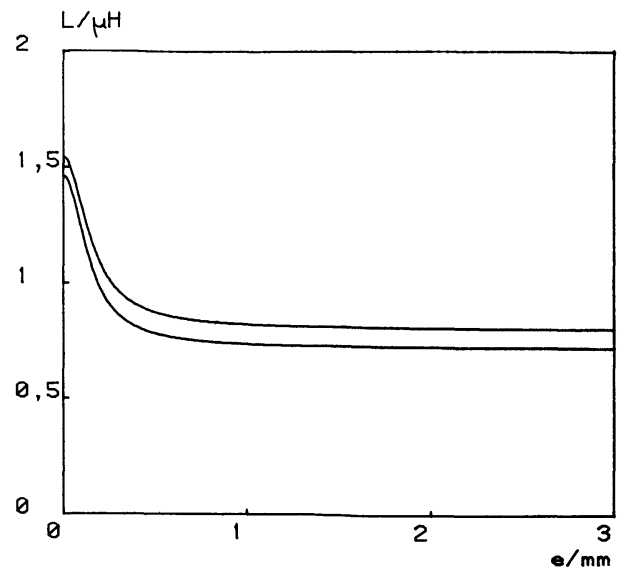

Fig. 16. - Inductance équivalente calculée avec $R_{2}=a+b / e$, $L_{2}=$ Cte, $M=$ Cte.

[Equivalent inductance calculated with $R_{2}=a+b / e, L_{2}=$ Cte, $M=$ Cte.]

$\mathrm{MLT}^{-1}$ en ordonnée. On pourra cependant avoir une valeur approchée de la pression en prenant $S=160 \mathrm{~cm}^{2}$. La courbe en trait plein de la figure 3 a été calculée à partir des mesures électriques (Figs. 6, 7, 8 et 9) et des formules (2.12) et (2.14) pour l'énergie $2 \mathrm{~kJ}$ du générateur « $6 \mathrm{~kJ}$ ». Elle s'accorde bien avec les points expérimentaux obtenus depuis $0,015 \mathrm{~mm}$. Les courbes calculées correspondant aux autres énergies, se déduisent de la courbe à $2 \mathrm{~kJ}$ par affinité ; on ne les a pas reproduites pour ne pas alourdir la figure.

Pour le cuivre et le laiton les valeurs de $I_{0}, \omega$ et $\tau$ sont voisines de celles de l'aluminium (se référer par exemple à $L(d)$, Fig. 9) et la courbe théorique par conséquent peu différente. Ne disposant pas d'échantillons très minces pour ces métaux, il n'a pas été utile d'effectuer la comparaison pour $e>0,5 \mathrm{~mm}$, domaine où les paramètres électriques varient peu. Notons simplement que $I_{0}$ est un peu plus faible pour le laiton que pour le cuivre et l'aluminium, ce qui est confirmé par le rapprochement de la figure 5 avec les figures 3 et 4 .

5.3 Déformations. - En fonction de l'énergie du générateur, les déformations obtenues varient linéairement pour toutes les épaisseurs et pour chaque métal (Figs. 10, 11 et 12). Ce résultat est pratique en vue d'applications car on détermine aisément l'énergie nécessaire pour obtenir une déformation donnée :

$$
E=E_{0}+K\left(e-e_{0}\right) .
$$

Le tableau I rassemble un certain nombre de valeurs de $E_{0}, K$ et $e_{0}$ pour les trois métaux étudiés.

Les mesures précédentes ont été faites avec une bobine de notre fabrication (diamètre utile $160 \mathrm{~mm}$ ) et une matrice annulaire de $100 \mathrm{~mm}$ de diamètre intérieur.

Ce type de bobine résulte de nombreux essais et peut être considéré comme optimisé au point de vue nombre de spires, rapport entre largeurs des spires de conducteur et d'isolant. Des résultats pour d'autres dimensions demanderaient la construction de bobines de différentes tailles, et leur optimisation afin d'obtenir des rendements comparables.

6. Conclusion. - Le pendule balistique que nous avons réalisé s'est révélé bien adapté à la mesure des efforts exercés par une impulsion de champ magnétique sur une plaque métallique. D'un autre côté, l'enregistrement du transitoire électrique a permis la détermination de l'amplitude, de la fréquence et de la constante de temps de $I(t)$ nécessaires à l'évaluation calculée de $P(t)$ à diverses épaisseurs. L'accord avec les mesures au pendule est satisfaisant.

Faute d'une détermination locale de la distribution des courants de Foucault dans l'échantillon, c'est l'inductance de l'ensemble bobine-plaque, vu du générateur, qui représente les différences de comportement en fonction de l'épaisseur ou d'un métal à l'autre. Fixant les valeurs de $I_{0}, \omega$ et $\tau$, elle détermine en effet $P(t)$.

Les déformations engendrées par ces impulsions sont fonction linéaire de l'énergie électrique dépensée pour les produire. Cette facilité est appréciable pour le concepteur d'une installation de mise en forme.

7. Remerciements. - Les auteurs expriment leur gratitude à J. Y. Renaud, M. Poirier, D. Priem pour leur participation à ce travail.

\section{ANNEXE}

Résolution de l'équation de diffusion de $\boldsymbol{H}$ avec une condition à la limite sinusoïdale amortie. - Soit $\frac{\partial^{2} H}{\partial x^{2}}=\mu \sigma \frac{\partial H}{\partial t}$ l'équation. Posons $H(x, t)=X(x) . T(t)$

$$
\frac{X^{\prime \prime}}{X}=\mu \sigma \frac{T^{\prime}}{T}=\alpha^{2} \quad \text { (complexe) }
$$

\begin{tabular}{|c|c|c|c|c|c|c|c|c|c|c|}
\hline & \multicolumn{3}{|c|}{ Aluminium } & \multicolumn{3}{|c|}{ Cuivre } & \multicolumn{3}{|c|}{ Laiton } & \\
\hline$E_{0}$ & & 1 & & & 0,5 & & & 0,4 & & $\mathrm{~kJ}$ \\
\hline$e_{0}$ & & 0,1 & & & 0,2 & & & 0,2 & & $\mathrm{~mm}$ \\
\hline$\varepsilon$ & 0,05 & 0,1 & 0,15 & 0,01 & 0,03 & 0,05 & 0,01 & 0,03 & 0,05 & \\
\hline$K$ & 1,8 & 2,8 & 4,1 & 2 & 5,5 & 7,8 & 2,4 & 5,4 & 8,2 & $\mathrm{~kJ} / \mathrm{mm}$ \\
\hline
\end{tabular}

Tableau I. - Coefficients permettant de calculer l'énergie nécessaire à une déformation donnée. 
$X^{\prime \prime}=\alpha^{2} X$ s'intègre en $X=A \mathrm{e}^{-\alpha x}$ (seul le signe est physique)

$T^{\prime}=\frac{\alpha^{2}}{\mu \sigma} T$ s'intègre en $T=B \exp \left(\alpha^{2} / \mu \sigma\right) t$

d'où une solution :

$$
H_{1}=A_{1} B_{1} \mathrm{e}^{-\alpha x} \exp \left(\alpha^{2} / \mu \sigma\right) t .
$$

On aurait de même :

$$
H_{2}=A_{2} B_{2} \mathrm{e}^{-\beta x} \exp \left(\beta^{2} / \mu \sigma\right) t
$$

$H_{1}+H_{2}$ est aussi solution. C'est sur elle qu'on va appliquer la condition à la limite

$$
H(0, t)=H_{0} \mathrm{e}^{-t / \tau} \sin \omega t .
$$

Par identification, on obtient :

$$
\alpha^{2}=\mu \sigma \omega\left(i-\frac{1}{\omega \tau}\right) \quad \beta^{2}=-\mu \sigma \omega\left(i+\frac{1}{\omega \tau}\right)
$$

$$
A_{1} B_{1}=-A_{2} B_{2}=\frac{1}{2 i}
$$

et si l'on pose $\alpha=\chi+i \eta$

$$
\begin{aligned}
& \chi=\left[\frac{\mu \sigma \omega}{2}\left(\left(1+\frac{1}{\omega^{2} \tau^{2}}\right)^{1 / 2}-\frac{1}{\omega \tau}\right)\right]^{1 / 2} \\
& \eta=\left[\frac{\mu \sigma \omega}{2}\left(\left(1+\frac{1}{\omega^{2} \tau^{2}}\right)^{1 / 2}+\frac{1}{\omega \tau}\right)\right]^{1 / 2}
\end{aligned}
$$

et

$$
\beta=\chi-i \eta
$$

alors

$$
H=H_{1}+H_{2}=H_{0} \mathrm{e}^{-t / \tau} \mathrm{e}^{-\chi x} \sin (\omega t-\eta x)
$$

ce qui montre que le champ se propage en s'affaiblissant avec une vitesse $\omega / \eta \cdot \chi^{-1}$ est l'épaisseur de peau en régime amorti. Elle diffère peu de la valeur $\left(\frac{2}{\mu \sigma \omega}\right)^{1 / 2}$ si $\omega \tau>1(5 \%$ pour $\omega \tau=10)$.

\section{Bibliographie}

[1] Brower, D. F., " Magnetic pulse forming ", présenté à Automot. Eng. Congress, Detroit (1962).

[2] Leroy, M., Thèse de Doctorat ès Sciences, Nantes (1972).

[3] HaRding, J., Edit., Mechanical properties at high rates of strain, (The Institute of Physics) 1974.

[4] LindHOLM, U. S., Edit., Mechanical behavior of Materials under dynamic loads (New York) 1968.

[5] Renaud, J. Y., C. R. Hebd. Séan. Acad. Sci. B 281 (1975) 89.

[6] J JNSEN, H., IEEE Trans. Ind. Gen. Appl. no 4 juillet/août (1968).
[7] Fluerasu, C., Rev. Roum. Sci. Tech. 15 (1970) 457.

[8] LaL, G. K. et Hillier, M. J., Int. J. Mech. Sci. 10 (1968) 491.

[9] Basu, S. et Srivastava, K. D., IEEE Trans. Power Appar. Syst. 88, no 8 (1969)

[10] Fruhling, A., Cours d' Electricité 2196.

[11] Sмith, W. E., Br. J. Appl. Phys., 16, no 3 (1965).

[12] Chéron, R. et Renaud, J. Y., Rev. Fr. Méc. 77 (1981).

[13] Tobe, T., Kato, M. et Obara, H., Bull. JSME $22 \mathrm{n}^{\circ} 164$ (1979). 